EPJ Web of Conferences 81, 03006 (2014)

DOI: $10.1051 /$ epjconf/20148103006

(C) Owned by the authors, published by EDP Sciences, 2014

\title{
Searching a dark photon with HADES
}

\author{
M.Gumberidze ${ }^{1, a}$ and R.Holzmann ${ }^{2}$ for the HADES Collaboration \\ ${ }^{1}$ Technische Universität Darmstadt, 64289 Darmstadt, Germany \\ ${ }^{2}$ GSI Helmholtzzentrum für Schwerionenforschung GmbH, 64291 Darmstadt, Germany
}

\begin{abstract}
The existence of a photon-like massive particle, the $\gamma^{\prime}$ or dark photon, is postulated in several extensions of the Standard Model to explain some recent puzzling astrophysical observations, as well as to solve the sofar unexplained deviation between the measured and calculated values of the muon anomaly. The dark photon, unlike the conventional photon, would have mass and would be detectable via its mixing with the latter. We present a search for the $e^{+} e^{-}$decay of such a hypothetical particle, also named $\mathrm{U}$ vector boson, in inclusive dielectron spectra measured by HADES in the $\mathrm{p}(3.5 \mathrm{GeV})+\mathrm{p}$, $\mathrm{Nb}$ reactions, as well as the $\mathrm{Ar}(1.756 \mathrm{GeV} / \mathrm{u})+\mathrm{KCl}$ reaction. An upper limit on the kinetic mixing parameter squared $\left(\epsilon^{2}\right)$ at $90 \%$ CL has been obtained for the mass range $\mathrm{M}(\mathrm{U})=0.02-0.55 \mathrm{GeV} / \mathrm{c}^{2}$ and is compared with the present world data set. Furthermore, an improved upper limit of $2.3 \times 10^{-6}$ at $90 \%$ CL has been set on the branching ratio of the helicity-suppressed direct decay of the $\eta$ meson $\eta \rightarrow e^{+} e^{-}$.
\end{abstract}

\section{Introduction}

Observations of the cosmic electron and/or positron flux by ATIC [1], PAMELA [2], HESS [3], Fermi [4], and recently the AMS02 collaboration [5] have revealed an unexpected excess at momenta above $10 \mathrm{GeV} / c$, in particular in the positron fraction $e^{+} /\left(e^{-}+e^{+}\right)$. These observations cannot easily be reconciled in a consistent way with known astrophysical sources [6] and alternative theoretical explanations have therefore been put forward. In particular, scenarios in which the excess radiation stems from the annihilation of weakly interacting dark matter particles $[6,7]$ might offer an enticing solution to this puzzle.

To accommodate DM in elementary particle theory and to allow it to interact with visible matter, it has been proposed to supplement the Standard Model (SM) with an additional sector characterized by another $U(1)^{\prime}$ gauge symmetry [9-11]. The corresponding vector gauge boson — called $U$ boson, $A^{\prime}, \gamma^{\prime}$, or simply dark photon - would thereby mediate the annihilation of DM particles into charged lepton pairs. Indeed, from theoretical arguments a kinetic mixing of the $U(1)^{\prime}$ and $U(1)$ symmetry groups would follow [12], providing a natural connection between the dark and SM sectors. For that purpose, a mixing parameter $\epsilon$ has been introduced [9] relating the respective coupling strengths $\alpha^{\text {c }}$ and $\alpha$ of the dark and SM photons to visible matter via $\epsilon^{2}=\alpha^{\prime} / \alpha$.

Through the $U(1)-U(1)^{\prime}$ mixing term the $U$ boson would be involved in all processes which include real or virtual photons [13]. On the other hand, any search for a $U$ boson will have to deal

a e-mail: m.gumberidze@gsi.de 
with the large unavoidable background from standard QED radiative processes [14], namely meson and baryon electromagnetic decay leading to lepton pairs.

\section{The HADES experiment}

The high-acceptance dielectron spectrometer HADES operates at the GSI Helmholtzzentrum für Schwerionenforschung in Darmstadt, where it uses the beams from the heavy-ion synchrotron SIS18 in the few-GeV beam-energy range. A detailed description of the set-up can be found in [15].

In the experiments discussed here a proton beam with a kinetic energy of $\mathrm{E}_{p}=3.5 \mathrm{GeV}$ and an average intensity of about $2 \times 10^{6}$ particles per second was used to bombard either a solid 12-fold segmented niobium target (with $2.8 \%$ nuclear interaction probability) [17] or a liquid hydrogen target ( $1 \%$ interaction probability) [16]. Likewise, a $1.76 \mathrm{GeV} / u$ Ar beam was used to bombard $\mathrm{KCl}$ targets.

In the data analysis, electrons and positrons were identified by applying selection cuts to the $\mathrm{RICH}$, pre-shower and energy-loss signals. The particle momenta were obtained by tracking the charged particles through the HADES magnetic field; the latter were combined two-by-two to fully reconstruct the 4-momentum of $e^{+} e^{-}$pairs. A detailed description of this analysis is given in $[15,18]$.

\section{The U-boson search}

The search for the $U$ boson can be performed with HADES using all electromagnetic decays typically populated in few-GeV hadronic interactions, that is mostly $\pi^{0} \rightarrow \gamma U, \eta \rightarrow \gamma U$, and $\Delta \rightarrow N U$, followed by $U \rightarrow e^{+} e^{-}$. In contrast to previous experiments [21, 22] focusing on a specific decay channel, our search was based on the inclusive measurement of all $e^{+} e^{-}$pairs produced in a given mass range. Because of the expected long lifetime of the $U$ boson the width of its signal is determined by the detector resolution.

The present analysis is based on the raw dilepton mass spectra, i.e. spectra not corrected for efficiency and acceptance. The low invariant-mass region of the spectra $\left(\mathrm{M}_{e e}<0.13 \mathrm{GeV} / c^{2}\right)$ is dominated by $\pi^{0}$ Dalitz decays, at intermediate masses $\left(0.13 \mathrm{GeV} / c^{2}<\mathrm{M}_{e e}<0.55 \mathrm{GeV} / c^{2}\right), \eta$ and $\Delta$ Dalitz decays prevail, and the high-mass region is populated mostly by low-energy tails of vector-meson decays $[16,17]$. However, as the electromagnetic decay branching ratios decrease with increasing particle mass, resulting in low sensitivity, we restrict our search to $M_{U}<0.6 \mathrm{GeV} / c^{2}$.

Our search for a narrow resonant state in the $e^{+} e^{-}$mass distributions has been conducted in the following way: (1) The $d N / d M_{e e}$ spectra, measured in either of the analyzed reactions, was fitted piece-wise with a model function consisting of a $5^{\text {th }}$-order polynomial and a Gauss peak of fixed position $M_{e e}$ and fixed width. (2) The adjustment was done by sliding a fit window of width $\pm 4 \sigma(M)$ over the spectrum in steps of $3 \mathrm{MeV} / \mathrm{c}^{2}$. In each step, the fit delivered a parameterization of the local background in presence of a possible Gaussian signal of given width $\sigma(M)$. (3) Consequently, a statistical likelihood-based test must be performed to determine at a given Confidence Level (CL) an upper limit (UL) for a possible $U$-boson signal [23]. In our case, background and $e^{+} e^{-}$efficiency corrections are needed to extract an absolute signal yield, and as both are known with limited accuracy only, we have used the method proposed by Rolke, Lopez and Conrad [24] to compute the UL at a confidence level $\mathrm{CL}=90 \%$.

(4) Finally, in Fig. 1 we show the HADES result together with a compilation of limits from the searches conducted by BaBar [25], KLOE-2 [22], APEX [20], WASA at COSY [21], and A1 at MAMI [19].

At low masses $\left(M_{U}<0.1 \mathrm{GeV} / c^{2}\right)$ we clearly improve on the recent result obtained by WASA [21], excluding now to a large degree the parameter range allowed by the muon $g-2$ anomaly. At 
higher masses, the sensitivity of our search is compatible with, albeit somewhat lower than the combined KLOE-2 analysis of $\phi$ decays. Our data probe, however, the $U$-boson coupling in $\eta$ decays and add hence complementary information. At masses above the $\eta$ mass, the inclusive dilepton spectrum is fed by $\Delta$ (and to some extent heavier baryon resonance) decays which offer only small sensitivity, partly due to the small electromagnetic branching ratio $\left(B R_{N \gamma} \simeq 10^{-3}-10^{-2}\right)$ and partly due to the decreasing $B R_{U \rightarrow e e}$ at high $M_{U}$. Recently the high-mass region has been covered by data from the BaBar experiment: $e^{+} e^{-} \rightarrow e^{+} e^{-}$and $e^{+} e^{-} \rightarrow \mu^{+} \mu^{-}$([26] not shown on Fig. 1). Our results at low masses will be improved by including recent data from the $1.23 \mathrm{GeV} / u \mathrm{Au}+\mathrm{Au}$ reaction. We expect to lower the UL by a factor 2 .

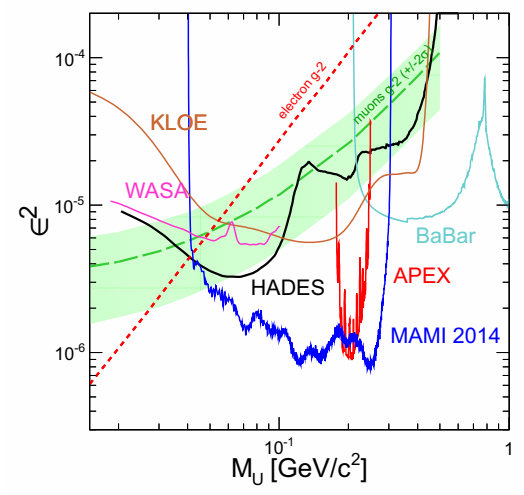

Figure 1. (Color online) The $90 \% \mathrm{Cl}$ upper limit on $\epsilon^{2}$ versus the U-boson mass obtained from the combined analysis of the HADES data (solid black curve) in comparison with existing limits from the MAMI/A1, APEX, BaBar, WASA, and KLOE-2 experiments. In addition the $g-2$ constrains are also plotted.

\section{$4 \mathrm{UL}$ on the rare decay $\eta \rightarrow \mathbf{e}^{+} \mathbf{e}^{-}$}

The direct decay of the $\eta$ meson into a lepton pair $\left(e^{+} e^{-}\right.$or $\left.\mu^{+} \mu^{-}\right)$can only proceed through a 2photon intermediate state. The $e^{+} e^{-}$decay is furthermore strongly suppressed by helicity conservation. Calculations based on chiral perturbation theory and quark models put its branching ratio at $B R_{\eta \rightarrow e^{+} e^{-}}^{Q C D} \simeq 5 \times 10^{-9}$. The present analysis of our $p+\mathrm{Nb}$ data allows to set an improved experimental limit $(\mathrm{CL}=90 \%)$ at $2.5 \times 10^{-6}$. Combining the $p+\mathrm{p}$ and $p+\mathrm{Nb}$ results, a final limit of $2.3 \times 10^{-6}$ can be given (see Fig. 2), i.e. about a factor 2.5 lower than the present PDG value [8], but still a far way above theoretical predictions [27].

\section{Acknowledgements}

The HADES Collaboration gratefully acknowledges the support by BMBF grants 06DR9059D, 05P12CRGHE, 06FY171, 06MT238 T5, and 06MT9156 TP5, by HGF VH-NG-330, by DFG EClust 153, by GSI TMKRUE, by the Hessian LOEWE initiative through HIC for FAIR (Germany), by EMMI GSI, TU Darmstadt (Germany): VHNG-823, Helmholtz Alliance HA216/EMMI, by grant GA CR 13-067595 (Czech Rep.), by grant NN202198639 (Poland), Grant UCY/3411-23100 (Cyprus),by CNRS/IN2P3 (France), by INFN (Italy), and by EU contracts RII3-CT-2005-515876 and HP2 227431.

\section{References}

[1] J. Chang et al., Nature 456 (2008) 362. 


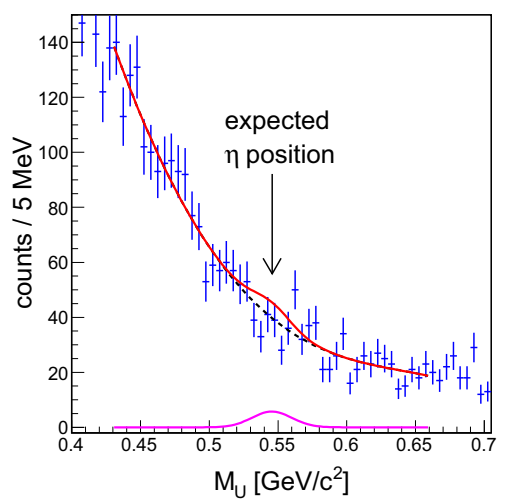

Figure 2. (Color online) Zoom into the $\eta$ peak region of the invariant-mass distribution of $e^{+} e^{-}$pairs reconstructed in the $\mathrm{p}(3.5 \mathrm{GeV})+\mathrm{Nb}$ reaction. The data is fitted with a polynomial (dashed black curve) onto which is a gaussian signal of strength $\mathrm{S}$ set equal to the found upper limit ( $\mathrm{CL}=90 \%)$ of $\mathrm{BR}_{\eta \rightarrow e^{+} e^{-}}<2.5 \times 10^{-6}$ (solid red and pink curves).

[2] O. Adriani et al., Nature 458 (2009) 607.

[3] F. Aharonian et al., Astron. Astrophys. 508 (2009) 561.

[4] A. Abdo et al., Phys. Rev. Lett. 102 (2009) 181101.

[5] L. Accardo et al., Phys. Rev. Lett. 113 (2014) 121101.

[6] I. Cholis, L. Goodenough, N. Weiner, Phys. Rev. D 79 (2009) 123505.

[7] I. Cholis et al., Phys. Rev. D 80 (2009) 123518.

[8] J. Behringer et al. (Particle Data Group), Phys. Rev. D 86 (2012) 010001.

[9] P. Fayet, Phys. Lett. B 95 (1980) 285.

[10] C. Boehm, P. Fayet, Nucl. Phys. B 683 (2004) 219.

[11] M. Pospelov, A. Ritz, M. B. Voloshin, Phys. Lett. B 662 (2008) 53.

[12] B. Holdom, Phys. Lett. B 166 (1986) 196.

[13] M. Reece, L.-T. Wang, JHEP 0907 (2009) 051.

[14] L. Landsberg, Phys. Rept. 128 (1985) 301.

[15] G. Agakishiev et al. (HADES Collaboration), Eur. Phys. J. A 41 (2009) 243.

[16] G. Agakishiev et al. (HADES Collaboration), Eur. Phys. J. A 48 (2012) 64.

[17] G. Agakishiev et al. (HADES Collaboration), Phys. Lett. B 715 (2012) 304.

[18] G. Agakishiev et al. (HADES Collaboration), Phys. Rev. C 84 (2011) 014902.

[19] H. Merkel et al. (A1 Collaboration), Phys. Rev. Lett. 112 (2014) 221802.

[20] S. Abrahamyan et al. (APEX Collaboration), Phys. Rev. Lett. 107 (2011) 191804.

[21] P. Adlarson et al. (WASA-at-COSY Collaboration), Phys. Lett. B 726 (2013) 187.

[22] D. Babuski et al. (KLOE-2 Collaboration), Phys. Lett. B 720 (2013) 111.

[23] G. Cowan, K. Cranmer, E. Gross, O. Vitells, Eur. Phys. J. C 71 (2011) 1554.

[24] W.A. Rolke, A.M. Lopez, J. Conrad, Nucl. Inst. Meth. Phys. Res. A 551 (2005) 493.

[25] B. Aubert et al. (BaBar Collaboration), arXiv:0902.2176.

[26] J. P. Lees et al. (BaBar Collaboration), arXiv:1406.2980

[27] A.E. Dorokhov, M.A. Ivanov, Phys. Rev. D 75 (2007) 114007. 